\title{
Specularity Detection Using Time-of-Flight Cameras
}

\author{
Faisal Mufti ${ }^{1}$ and Robert Mahony ${ }^{2}$ \\ 1 Center for Advanced Studies in Engineering \\ faisal.mufti@ieee.org \\ 2 Australian National University \\ robert. mahony@anu. edu. au
}

\begin{abstract}
Time-of-flight (TOF) cameras are primarily used for range estimation by illuminating the scene through a TOF infrared source. However, additional background sources of illumination of the scene are also captured in the measurement process. This paper exploits conventional Lambertian and Phong's illumination models, developed for $2 \mathrm{D}$ CCD image cameras, to propose a radiometric model for a generic TOF camera. The model is used as the basis for a novel specularity detection algorithm. The proposed model is experimentally verified using real data.
\end{abstract}

Keywords: Time-of-flight, Radiometric Modelling, Specularity Detection, Reflectance Modelling.

\section{Introduction}

Objects and materials in real world appear differently to an observer depending on the nature of the light source that they are illuminated by and the manner in which the light is reflected to the observer. Computer vision [1 and computer graphics 2] researchers have extensively treated reflectance modelling for image analysis, rendering and scene geometry. Specular highlights can be used to provide information about the surface [12 and the illumination geometry [14] in a natural scene. However, saturation effects, due to specularity in intensity images, often create problems for image processing algorithms in real environments [13]. In addition, many computer vision algorithms [7, 9] are dependent on surface illumination of an object and changing illumination conditions, such as highly saturated highlights interfere and adversely effect the camera image. It is therefore, important to detect specular highlights in image processing applications and algorithms. Since photometric understanding (using 2D CCD camera technology) of illumination modelling is focused on intensity, specularity detection methods 8, 1 are normally based on chromaticity of the region.

3D time-of-flight (TOF) cameras provide information in addition to intensity that can be incorporated in a reflectance model. A TOF camera works on the principle of measuring time of flight of a modulated infrared light signal as phase offset after reflection and provides amplitude, phase and intensity data over a full image array at video frame rate $[5]$. 
This paper presents a novel algorithm for specularity detection using TOF cameras. In the proposed radiometric framework, the background light sources and the dependencies between amplitude, intensity and phase/range measurements of a TOF camera are exploited. The model is utilized for specularity detection using real TOF camera data.

\section{Reflectance Model}

Time-of-flight (TOF) sensors estimate distance to a target using the time of flight of a modulated infrared (IR) wave between the target and the camera. The sensor illuminates/irradiates the scene with a modulated signal of amplitude $A$ (exitance) and receives back a signal (radiosity) after reflection from the scene with background signal offset $I_{o}$ that includes non-modulated DC offset generated by TOF camera as well as ambient light reflected from the scene. The amplitude, intensity offset $I$ and phase of a modulated signal can be extracted by demodulating the incoming signal $A_{i}=A \cos \left(\omega t_{i}+\varphi\right)+I ;\left(t_{i}=i \cdot \frac{\pi}{2 \omega}, i=0, \ldots 3\right)[5]$. With known phase $\varphi$, modulation frequency $f_{\bmod }$ and precise knowledge of speed of light $c$, it is possible to measure the un-ambiguous distance $r$ from the camera [1].

The measurement parameters of amplitude $A$, intensity $I$, and range $r$ are not independent but depend on the reflectance characteristics of the scene 11 . In this discussion a near-field IR point source for the camera's active LED array, an ambient illumination and a far-field source for background illumination is considered. The primary source of illumination in TOF cameras is an IR source that produces a modulated IR signal offset and a non-modulated DC signal. Based on Phong's illumination model 14, 3, p. 729], the following discussion incorporates diffuse $(.)_{d}$, ambient $(.)_{a}$, and specular $(.)_{s}$ components of illumination.

\subsection{Modulated IR Source}

Let $P$ be a Lambertian surface in space with $n_{p}$ denoting the normal to each point $p \in P$ on the surface as shown in Figure. 1. Following the laws of radiometry [15] the amplitude of total radiance $A_{d}(p)$ (called radiosity) leaving point $p$ due to illumination by the modulated signal $A(s)$ is proportional to the diffuse reflectance or albedo $\rho_{d}(p)$ scaled by the cosine of arrival angle $\theta_{p}$ [10, p.68]. In the present analysis, the LED point sources of the camera are part of the compact IR array of the TOF camera, and can be approximated by a single virtual modulated point source [4, p. 78] with the centre of illumination aligned with the optical axis of the camera [6]. In this case, the integration for illuminating sources can be written as a function of the exitance of a single point source at $S$ as [4, p. 77] [1]

$$
A_{d}(p):=\frac{1}{\pi} \rho_{d}(p) \frac{A(s) \cos \theta_{p} \cos \theta_{s}}{r^{2}} .
$$

The irradiance of an image point $x$ is obtained [10, p. 48] as

$$
A_{d}(x)=\Upsilon A_{d}(p),
$$




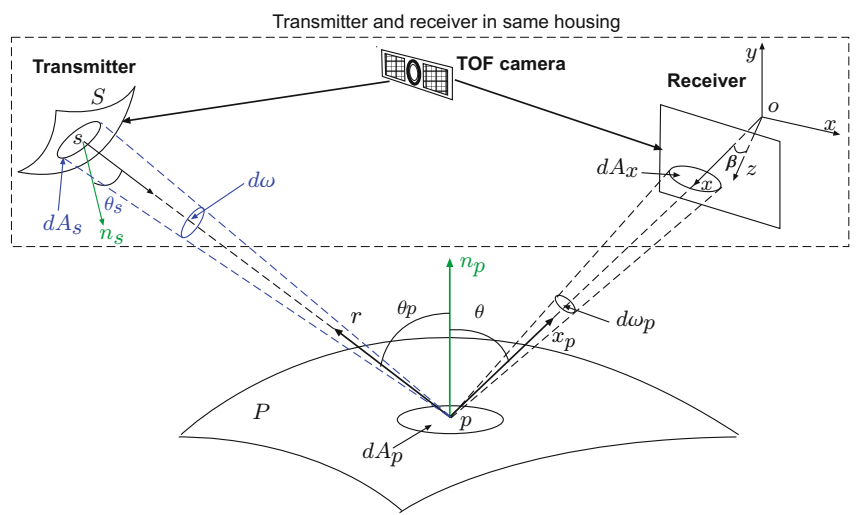

Fig. 1. Geometry of reflectance model for time-of-camera. Note that although the LED source and receiver of a physical TOF camera are co-located, it is difficult to provide a visualisation of this geometry. Here the source is shown separately to make is easier to see notation. However, in practice the directional vectors $r$ and $x_{p}$ are equal. Note that time variation (discussed in Section 2) of $A(s)$ does not need to be modelled as only the relative magnitude of $A(s)$ is of interest.

where $\Upsilon:=\Upsilon(x) \sqrt{1}$ is the lens collection [15] representing the vignetting due to aperture size and irradiance fall-off with cosine-fourth law.

\subsection{Non-modulated IR Source}

The TOF camera IR source produces a DC signal from the same IR source LEDs. This signal will have the same reflectance model as has been derived for the modulated IR source (see (11) ). The received signal $I_{c_{d}}(x)$ is given by [1]

$$
I_{c_{d}}(x)=\Upsilon I_{c_{d}}(p) \text {. }
$$

The effect of this signal is an added offset to the modulated signal that provides better illumination of the scene.

\subsection{Far-Field Background Illumination}

For a point source $q \in Q$ that is far away compared to the area of the target surface, the exitance $I_{b_{d}}(q)$, does not depend on the distance from the source or the direction in which the light is emitted. Such a point source can be treated as constant [4, p. 76]. The radiosity perceived by a TOF image plane as a result of this IR source is given by [1]

$$
\begin{aligned}
I_{b_{d}}(x) & =\frac{\Upsilon}{\pi} \rho_{d}(p) I_{b}(q) \cos \theta_{q} \\
& =\Upsilon I_{b_{d}}(p) .
\end{aligned}
$$

where $\theta_{q}$ is the angle between normal to the surface point $p$.

\footnotetext{
$1:=$ Defination of a symbol
} 


\subsection{Ambient Background Illumination}

Consider an ambient background illumination of the scene i.e an illumination that is constant for the environment [4, p. 79] and produces a diffuse uniform lighting over the object [3, p. 273]. Let $I_{a}$ be the intensity (called exitance) of the ambient illumination, then the received intensity $I_{a}(p)$ from a point $p$ is expressed in an image plane as [1]

$$
\begin{aligned}
I_{a}(x) & =\frac{\Upsilon}{\pi} \rho_{a}(p) I_{a} \\
& =\Upsilon I_{a}(p),
\end{aligned}
$$

where $\rho_{a}$ is the ambient reflection coefficient which is often estimated empirically instead of relating it to the properties of a real material [3, p. 723].

\subsection{Specular Illumination}

Specular reflection is observed from a shiny surface when light is reflected in a single direction where the angle of incidence $\theta_{p}$ and angle of reflection $\theta$ are equal around the normal to the surface. The fall off effect of specular reflectance from shiny surfaces is modelled by $\cos ^{n} \alpha$, where $n$ is the specular reflection exponent [3] and $\alpha$ is the angle between direction of reflection and the view point. For imperfect shiny surfaces, specular reflection is spread over an angle $\alpha$ around the direct reflection. The received illumination components for intensity and amplitude observed in the image plane due to specularity are given by

$$
I_{(.)_{s}}(x):=\frac{\Upsilon \rho_{s}(p)}{\pi} \frac{I_{s}(s) \cos ^{n} \alpha \cos \theta_{s}}{r^{2}} ; A_{s}(x):=\frac{\Upsilon \rho_{s}(p)}{\pi} \frac{A_{s}(s) \cos ^{n} \alpha \cos \theta_{s}}{r^{2}}
$$

where the specular reflection coefficient $\rho_{s}(p)$, effects the brightness of specularity. Typical values of $n$ vary from 0 to several hundred depending upon the surface material. A value of 1 gives a broad fall off of specular reflectance and a high value results in sharp fall-off of the specular reflectance.

\section{Specularity Detection}

From the principles of TOF camera (see Section 2) signals one knows that intensity component of TOF carries information for both, amplitude of the modulated signal and the background offset $I_{o}$. The radiometric intensity measured by a TOF camera is then

$$
I:=A+I_{o} .
$$

The background offset $I_{o}$ is composed of DC offset $I_{c}$, due to the DC component of the illumination by the TOF camera LED array and background illumination that are modelled by an ambient illumination $I_{a}$ and a background illumination $I_{b}$ due to an infrared far field source present in the environment such as the Sun or other light source. Indexing the point $p$ in the scene by the TOF receiving pixel $x$, one has 


$$
I_{o}(x)=I_{a}(x)+I_{b_{d}}(x)+I_{b_{s}}(x)+I_{c_{d}}(x)+I_{c_{s}}(x),
$$

Using the total intensity of the TOF camera (7) and background offset (8) and dividing it by the total (diffuse plus specular) received amplitude, one has

$$
\frac{I(x)}{A(x)}=1+\frac{I_{a}(x)}{A(x)}+\frac{I_{b}(x)}{A(x)}+\frac{I_{c}(x)}{A(x)},
$$

where $I_{b}(x)=I_{b_{d}}(x)+I_{b_{s}}(x), I_{c}(x)=I_{c_{d}}(x)+I_{c_{s}}(x)$ and $A(x)=A_{d}(x)+A_{s}(x)$. Using the reflectance models derived earlier (2) (3), and the specular model (6), it is now possible to re-arrange (9) as

$$
\frac{I(x)}{A(x)}=1+\kappa_{c}(x)+\kappa_{a} \frac{r^{2}(x) \rho_{a}(p)}{\cos \theta_{s}\left[\rho_{d}(p) \cos \theta_{p}+\rho_{s}(p) \cos ^{n} \alpha\right]},
$$

where $\theta_{s}:=\theta_{s}(x)$ is a known function of a pixel and $\kappa_{a}$ is defined as the ratio of background ambient light $I_{a}$ to modulated TOF IR source $A(s)$. Observe that $\kappa_{a}$ does not depend upon scene or camera geometry and hence is a constant parameter over the full image array. Also for an indoor environment (such as the one with no direct sunlight effect) the terms involving $I_{b}$ in (9) is ignored in order to simplify the model and only ambient illumination (due to indoor lighting) component $I_{a}$ is considered. The parameter $\kappa_{c}=\kappa_{c}(x)$, is defined as the ratio of TOF non-modulated IR source $I_{c}(s)$ to TOF modulated IR source $A(s)$. Since the two sources of illumination originating from the TOF camera IR LED source have the same ray geometry, they are in direct proportion where $\kappa_{c}(x)$ is a camera based pixel $x \in \mathbb{R}^{2}$ parameter independent of the scene for an entire image [1].

Thus for each pixel $x$, one can re-write (10) as

$$
\kappa_{a}(x) \frac{\rho_{a}(p)}{\left[\rho_{d}(p) \cos \theta_{p}+\rho_{s}(p) \cos ^{n} \alpha\right]}:=\left(\frac{I(x)}{A(x)}-\kappa_{c}(x)-1\right) \frac{\cos \theta_{s}}{r^{2}(x)} .
$$

Define the specular measurement criterion $\check{\kappa}_{a}(x)$ based on measurements taken from the camera at a given time as

$$
\check{\kappa}_{a}(x) \frac{\rho_{a}(p)}{\left[\rho_{d}(p) \cos \theta_{p}+\rho_{s}(p) \cos ^{n} \alpha\right]}:=\left(\frac{\check{I}(x)}{\check{A}(x)}-\hat{\kappa}_{c}(x)-1\right) \frac{\cos \theta_{s}}{\check{r}^{2}},
$$

where $\hat{\kappa}_{c}(x) \in \mathbb{R}^{2}$ is an estimate of camera based pixel parameter for an entire image. Since $\kappa_{c}(x)$ is scene independent, it can be measured offline as $\hat{\kappa}_{c}(x)$ in a set of calibration experiments.

For a TOF camera, the light direction of the source and the receiver are collinear as a result specularity is only observed in the direction of IR light from the camera. For maximum specularity $\alpha=0$, the angle $\cos \theta_{p}=1$ and the left hand side of (12) is scaled by a constant term $C_{\rho}$ as

$$
\check{\kappa}_{a}(x) C_{\rho}:=\left(\frac{\check{I}(x)}{\check{A}(x)}-\hat{\kappa}_{c}(x)-1\right) \frac{\cos \theta_{s}}{\check{r}^{2}},
$$


where $C_{\rho}$ is given by

$$
C_{\rho}:=\frac{\rho_{a}(p)}{\rho_{d}(p)+\rho_{s}(p)} .
$$

Note that maximum specularity occurs when the angles $\left(\cos \theta_{p}, \cos ^{n} \alpha\right)$ have maximum values resulting in a higher denominator term with respect to numerator irrespective of the scaled constant terms of reflectivity coefficients $C_{\rho}$ (with decreasing denominator for non-perfect specularity regions). The specularity criterion can be easily formulated without explicit angle estimation. For a specular region, the TOF camera receives sufficient signal and the range data is reliable. As a result the specular radiometric criterion (13) has only one dominating parameter $\kappa_{a}$ representing the ratio of ambient offset to TOF IR amplitude. Consequently, specularity can be detected from a $\check{\kappa}_{a}(x)$ plot where

$$
\text { specularity }=\min \left|\check{\kappa}_{a}(x)\right| \forall(x)
$$

due to high IR amplitude signal and low background offset of intensity. A specular lobe around this point would be indicative of the surface material encoded by $n$.

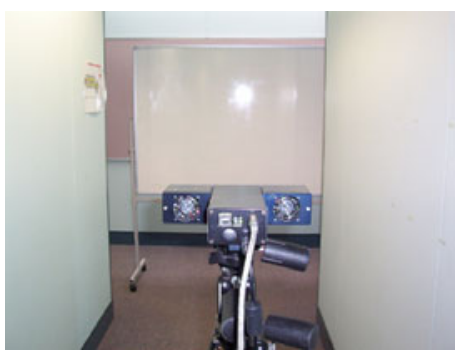

(a)

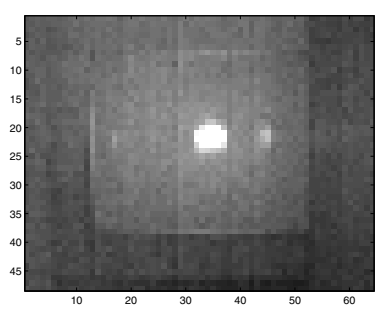

(b)

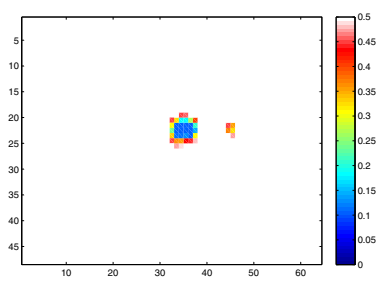

(c)

Fig. 2. (a) Picture taken from a normal CCD camera of the experimental setup showing TOF camera and a white board (b) Grayscale intensity image as observed in the TOF camera with specular lobe visible due to IR reflection from the board to camera. (c) Segmentation of specular and non-specular regions based on $\breve{\kappa}_{a}(x)$ of a frame. 


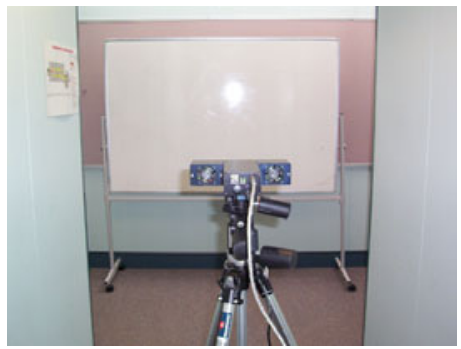

(a)

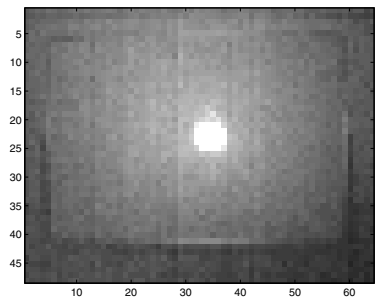

(b)

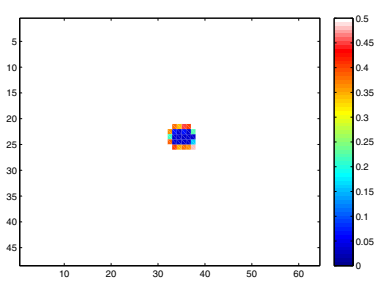

(c)

Fig. 3. (a) Picture taken from a normal CCD camera of the experimental setup (b) Intensity image as observed in TOF camera. (c) Segmentation of specular and nonspecular regions of a complete frame.

\section{Experiments}

An indoor environment was chosen for experiments as shown in Figure 2(a) and $3(\mathrm{a})$ using a white board placed in front of the camera. The board provided sufficient specular reflectance due to its surface material.

In the first case specular reflectance was picked up by the camera. The minimum point of $\breve{\kappa}_{a}(x)$ space represented the point of maximum specularity with the fall-off forming a lobe of specularity due to the surface material. Since the camera was placed in corridor, a few side reflections from the wall on the board caused a secondary specular lobe as observed in Figure 2(a) and Figure 2(b). These were picked up by the algorithm along with the main specular lobe as illustrated in Figure 2(c). In another experimental setup, as shown in Figure 3(a), the algorithm has picked the specular reflection (see Figure $3(\mathrm{c})$ that was observed in Figure $3(\mathrm{~b})$.

\section{Conclusion}

Unlike conventional cameras where only a single parameter is measured as intensity, TOF camera measures three independent parameters of amplitude, intensity and phase. These measurements along with the illumination conditions of the 
environment facilitated in deriving a radiometric model for specular highlights in TOF cameras. The proposed framework proved robust and effective for specular highlights detection in imaging algorithms.

\section{References}

1. Angelopoulou, E.: Specular highlight detection based on the fresnel reflection coefficient. In: Proc. IEEE 11th International Conference on Computer Vision ICCV 2007, pp. 1-8 (2007)

2. Cook, R.L., Torrance, K.E.: A reflectance model for computer graphics. ACM. Trans. on Graphics 1(1), 7-24 (1982)

3. Foley, J.D., Da, A., Feiner, S.K., Hughes, J.F.: Computer Graphics: Principles and Practices. Addisin-Wesley Publishing Company, Inc., Reading (1997)

4. Forsyth, D.A., Ponce, J.: Computer Vision: A Modern Approach. Prentice Hall, Englewood Cliffs (2003)

5. Kahlmann, T., Remondino, F., Guillaume, S.: Range imaging technology: new developments and applications for people identification and tracking. In: Proc. SPIE-IS\&T Electronic Imaging, San Jose, CA, USA, vol. 6491 (January 2007)

6. Kuhnert, K.D., Stommel, M.: Fusion of stereo-camera and PMD-camera data for real-time suited precise 3D environment reconstruction. In: Proc. IEEE/RSJ Int. Conf. Intell. Robot. Systs. (2006)

7. Lee, Y.B., You, B.J., Lee, S.W.: A real-time color-based object tracking robust to irregular illumination variations. In: Proc. ICRA Robotics and Automation IEEE Int. Conf., vol. 2, pp. 1659-1664 (2001)

8. Lin, S., Lee, S.W.: Detection of specularity using stereo in color and polarization. In: Proc. 13th International Conference on Pattern Recognition, vol. 1, pp. 263-267 (1996)

9. Lin, S., Lee, S.W.: Using chromaticity distributions and eigenspace analysis for pose-, illumination-, and specularity-invariant recognition of 3d objects. In: Proc. IEEE Computer Society Conference on Computer Vision and Pattern Recognition, pp. 426-431 (1997)

10. Ma, Y., Soatto, S., Košecká, J., Sastry, S.S.: An Inviation to 3-D Vision: From Images to Geomtric Models. ch. 2. Springer, Heidelberg (2003)

11. Mufti, F., Mahony, R.: Radiometric range image filtering for time-of-flight cameras. In: Proc. Int. Conf. on Computer Vision Theory and Applications (VISAPP 2010), Angers, France, vol. 1, pp. 143-152 (May 2010)

12. Nehab, D., Weyrich, T., Rusinkiewicz, S.: Dense 3d reconstruction from specularity consistency. In: Proc. IEEE Conference on Computer Vision and Pattern Recognition CVPR 2008, pp. 1-8 (2008)

13. Park, J.B., Kak, A.C.: A truncated least squares approach to the detection of specular highlights in color images. In: Proc. IEEE International Conference on Robotics and Automation ICRA 2003, vol. 1, pp. 1397-1403 (2003)

14. Phong, B.T.: Illumination for computer generated pictures. Comm. ACM 18, 311$317(1975)$

15. Sillion, F.X., Puech, C.: Radiosity and Global Illumination. Morgan Kaufmann, San Francisco (1994) 